\title{
Is ERAS effective and safe in laparoscopic gastrectomy for gastric carcinoma? A meta-analysis
}

\author{
Ming-zhe Li ${ }^{\dagger}$, Wen-hui Wu${ }^{\dagger}$, Liang Li, Xue-fu Zhou, Heng-liang Zhu, Jian-feng Li and Yu-long He*
}

\begin{abstract}
Background: It is still unclear whether enhanced recovery after surgery is effective and safe in laparoscopic gastrectomy for gastric carcinoma.

Methods: Cochrane library databases, Medline, Embase, and Pubmed were searched from January 1, 1986, to December 31, 2016. Randomized controlled trials (RCTs) comparing fast-track recovery with conventional recovery strategies in laparoscopic radical gastrectomy for gastric carcinoma were included. The main outcomes measured were postoperative hospital stay, time to first flatus, hospital charge, and overall complication rate.

Results: Six RCTs with 400 patients were included in this study. Fast-track surgery has shorter postoperative hospital stays (weighted mean difference (WMD) $-2.65 ; 95 \% \mathrm{Cl},-4.01$ to $-1.29, z=3.82, P<0.01$ ) and less hospitalization expenditure (WMD $-523.43 ; 95 \% \mathrm{Cl},-799.79$ to $-247.06, z=3.71, P<0.01)$ than conventional recovery strategies. There was no significant difference with respect to duration to first flatus (WMD $-17.72 ; 95 \% \mathrm{Cl},-39.46-4.02, z=1$. $60, P=0.11)$ and complication rate (OR 1.57; $95 \% \mathrm{Cl}, 0.82-2.98, z=1.37, P=0.17)$.
\end{abstract}

Conclusions: Enhanced recovery after surgery is effective and safe and is thus recommended in laparoscopic radical gastrectomy for gastric carcinoma.

Keywords: Fast-track surgery, Gastric carcinoma, Laparoscopic, Meta-analysis

\section{Background}

Gastric carcinoma is the second leading cause of cancer related deaths and is also the fourth most common carcinoma in the world [1]. Surgery is the most effective approach for treatment of gastric carcinoma. Laparoscopic gastrectomy was increasingly being used for gastric carcinoma in recent years. Compared with conventional surgery, laparoscopic gastrectomy had many advantages such as improvement in quality of life and faster recovery [2-4].

The enhanced recovery after surgery (ERAS), also called fast-track surgery (FTS), was initiated by Kehlet et al. in 1995 [5]. ERAS was a multidisciplinary approach aiming to accelerate recovery, minimize hospital stay, and reduce hospitalization expenditure, without compromising

\footnotetext{
* Correspondence: gene_crystal@163.com

${ }^{\dagger}$ Equal contributors

Department of Gastrointestinal Surgery, The Seventh Affiliated Hospital, Sun Yat-sen University, No.628 Zhenyuan Road, Guangming new district, Shenzhen 518017, China

effectiveness and patient safety. Several randomized controlled trials (RCTs) and retrospective studies had documented the benefits and safety of FTS program implementation in laparoscopic radical gastrectomy for gastric carcinoma [6-12]. However, it was still unclear whether enhanced recovery after surgery is effective and safe in laparoscopic gastrectomy. Some studies comparing FTS and conventional recovery strategies in laparoscopic gastrectomy for gastric carcinoma gave conflicting results [13-18]. Therefore, the aim of this meta-analysis was to review the literature systematically and to investigate the safety and efficacy of FTS in laparoscopic gastrectomy for gastric carcinoma.

\section{Methods \\ Search and selection strategies}

This study was approved by the ethic committee of the Seventh Affiliated Hospital of Sun Yat-sen University. Two authors (Ming-zhe Li and Wen-hui Wu) performed 
PRISMA Flow Diagram

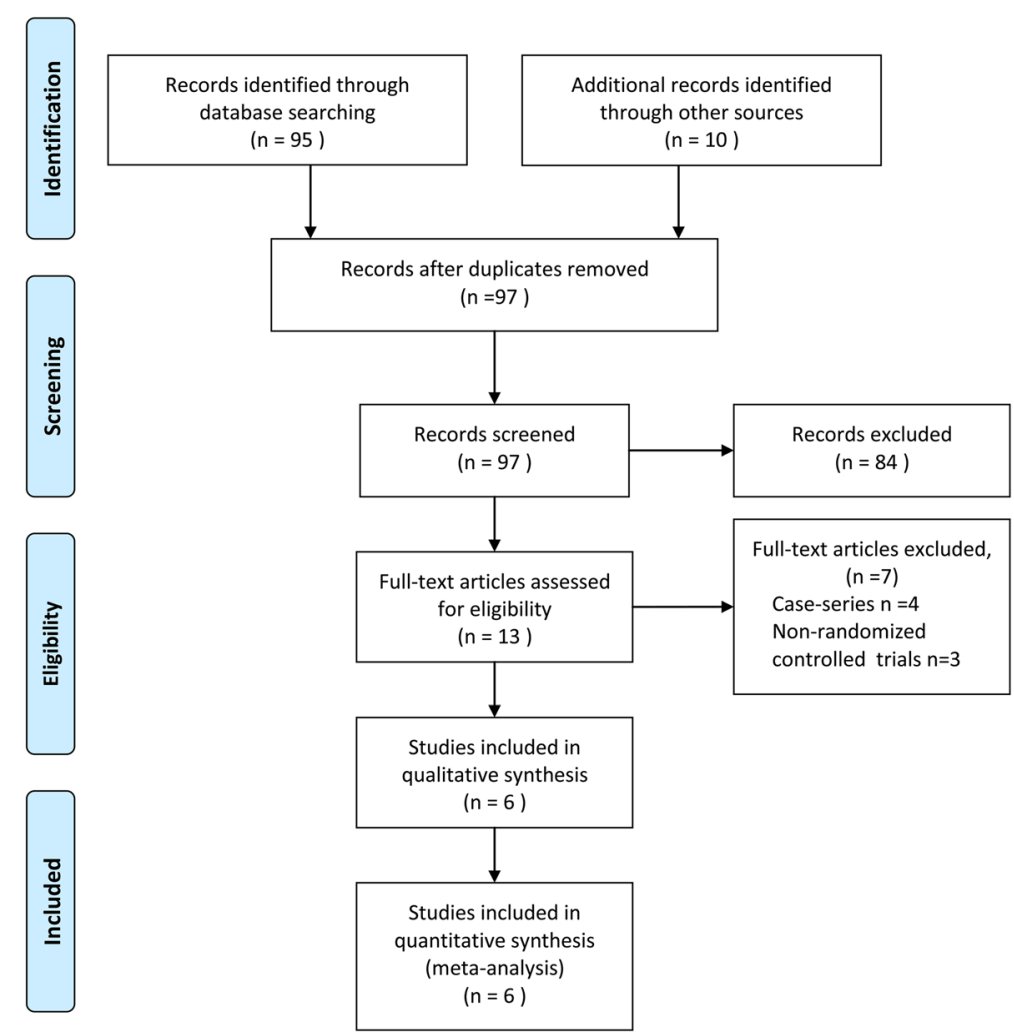

Fig. 1 Article selection flow chart

a systematic article search independently. Cochrane library databases, Medline, Embase, and Pubmed between January 1986 and December 2016 were electronically searched. The medical subject headings were as follows: "accelerated rehabilitation," "enhanced recovery," "fast track," "multimodal perioperative care," "laparoscopy," "laparoscopic," "minimally invasive," "gastrectomy," "gastr"," "gastric resection," and "stomach." The potentially relevant literature was retrieved through the consensus of the authors. The reference lists were cross-searched to identify additional literature. No language restriction was applied.

\section{Inclusion criteria and exclusion criteria}

Randomized clinical trials that compared fast-track surgery with conventional recovery strategies for patients undergoing laparoscopic gastrectomy and were published in full were included. Trials should report at least one relevant outcome (postoperative hospital

Table 1 Characteristics of studies included in this meta-analysis

\begin{tabular}{|c|c|c|c|c|c|c|}
\hline Author & Year & $\begin{array}{l}\text { Sample size } \\
\text { FTS/conventional }\end{array}$ & $\begin{array}{l}\text { Age (years) } \\
\text { FTS/conventional }\end{array}$ & $\begin{array}{l}\text { Gender ( } n, M: F) \\
\text { FTS/conventional }\end{array}$ & $\begin{array}{l}\mathrm{BMI}(\mathrm{kg} / \mathrm{m} 2) \\
\text { FTS/conventional }\end{array}$ & Type of surgery $(n)$ \\
\hline Hu et al. [13] & 2012 & $19 / 22$ & 59/62.5(median) & $(10: 9) /(10: 12)$ & $22.94 \pm 2.23 / 22.99 \pm 2.24$ & Distal gastrectomy(41) \\
\hline Kim et al. [14] & 2012 & $22 / 22$ & $52.64 / 57.45$ (mean) & $(13: 9) /(15: 7)$ & $23.40 \pm 3.17 / 23.77 \pm 3.54$ & Distal gastrectomy(44) \\
\hline Abdikarim et al. [15] & 2015 & $30 / 31$ & 63/62(median) & $(21: 9) /(20: 11)$ & NR & $\begin{array}{l}\text { Distal gastrectomy(44)/total } \\
\text { gastrectomy(17) }\end{array}$ \\
\hline Liu et al. [16] & 2016 & $21 / 21$ & 69.2/70.3(mean) & $(10: 11) /(12: 9)$ & $21.5 \pm 2.0 / 21.9 \pm 2.3$ & $\begin{array}{l}\text { Proximal gastrectomy } \\
\text { (10)/distal gastrectomy(21)/total } \\
\text { gastrectomy }(11)\end{array}$ \\
\hline Fang et al. [17] & 2016 & $33 / 30$ & 61.12/61.53(mean) & $(15: 18) /(16: 14)$ & NR & NR \\
\hline Xia et al. [18] & 2016 & 73/76 & 61/63(median) & $(48: 25) /(50: 26)$ & NR & NR \\
\hline
\end{tabular}


Table 2 Outcome variables

\begin{tabular}{lllll}
\hline Author & $\begin{array}{l}\text { Postoperative hospital stay (days) } \\
\text { FTS/conventional }\end{array}$ & $\begin{array}{l}\text { First flatus after surgery (h) } \\
\text { FTS/conventional }\end{array}$ & $\begin{array}{l}\text { Hospital charge (dollars) } \\
\text { FTS/conventional }\end{array}$ & $\begin{array}{l}\text { Complication N (\%) } \\
\text { FTS/conventional }\end{array}$ \\
\hline Hu et al. [13] & $7($ range 5.5-10)/7.5(range 6-11) & $58($ range 35-72)/65.5(range 35-72) & $4815.51 \pm 334.02 / 5212.72 \pm 447.26^{*}$ & $12(63.2) / 8(36.4)$ \\
Kim et al. [14] & $5.36 \pm 1.46 / 7.95 \pm 1.98^{*}$ & $63.05 \pm 18.62 / 67.41 \pm 15.28$ & $7454.3 \pm 705.8 / 7771.8 \pm 934.2$ & $3(13.6) / 4(18.2)$ \\
Abdikarim et al. [15] & $6.8 \pm 1.1 / 7.7 \pm 1.1^{*}$ & NR & NR & $1(3.3) / 2(6.5)$ \\
Liu et al. [16] & $6.3 \pm 1.5 / 7.8 \pm 1.8^{*}$ & $48 \pm 28.8 / 60 \pm 26.4$ & $4884.43 \pm 407.04 / 5625.82 \pm 276.2^{*}$ & $11(52.4) / 6(28.6)$ \\
Fang et al. [17] & $11.0 \pm 2.0 / 18.5 \pm 5.5^{*}$ & $60 \pm 12 / 96 \pm 24^{*}$ & NR & $2(7.1) / 2(6.5)$ \\
Xia et al. [18] & $6.38 \pm 2.04 / 8.62 \pm 2.87^{*}$ & NR & NR & $2(2.7) / 2(2.6)$ \\
\hline
\end{tabular}

FTS fast-track surgery, NR not reported

${ }^{*} P<0.05$. Italics indicate significance

stay, duration to first flatus after operation, hospitalization expenditure, and short-term complication).

Authors with more than one published literature were represented by the most recent publication. Non-randomized studies, cohort studies, retrospective studies, and other studies that did not fulfill the inclusion criteria were excluded. Because this study focused only on laparoscopic gastrectomy, completely open or hand-assisted surgery was not included.

\section{Outcome measures}

The outcome measures were postoperative hospital stay, duration to first flatus, hospital charge, and overall complication rate. Postoperative hospital stay was calculated from the date of surgery to the date of discharge.

\section{Method of review}

This study was prepared in accordance with the Preferred Reporting Items for Systematic Reviews and Meta-Analyses (PRISMA) statement [19]. Each article was independently reviewed by two researchers (Ming-zhe Li and Wen-hui $\mathrm{Wu}$ ) using the double method for eligibility by the inclusion criteria. Prior to final analysis, the article was confirmed by a third researcher ( $\mathrm{Yu}$-long $\mathrm{He}$ ) and all conflicts were discussed and resolved. The quality of the randomized clinical trials was independently assessed using Jadad's scoring system by two researchers (Ming-zhe $\mathrm{Li}$ and Wen-hui Wu) [20].

\section{Statistical analysis}

Statistical analyses were performed using RevMan 5.0 software (Cochrane Information Management System). For continuous variables (postoperative hospital stay, duration to first flatus after surgery, and hospital charge), weighted mean differences (WMDs) with 95\% confidence intervals (CIs) were calculated. For dichotomous variables (complication), odds ratios (ORs) with 95\% CIs were calculated. In accordance with the current exchange rate, the exchange rate of the renminbi (RMB) against the US dollar in hospitalization expense is 6.879:1. $I^{2}$ values were used to assess heterogeneity between articles. Publication bias was evaluated using funnel plots. If there was no significant heterogeneity among the studies, fixed effects model was used to pool studies. Otherwise, a random effects model was used. Level of statistical significance was set at $P<0.05$.

\section{Results}

Article search

One hundred and five articles were found by the literature search. Ninety-seven articles were selected for more detailed review after eliminating duplicates. Based on the inclusion criteria, 6 RCTs with 400 patients were included for meta-analysis (Fig. 1) [13-18]. Characteristics of each trial are shown in Table 1. Included literature was published from year 2012 to 2016. The number of patients in these studies ranged from 19 to 76 . A total of 198 patients were in the FTS group, and 202 patients were in the

Table 3 Jadad's score

\begin{tabular}{|c|c|c|c|c|c|c|}
\hline Author & Year & Randomization & Allocation concealment & Blinding & Withdrawal & Total Jadad score \\
\hline Hu et al. [13] & 2012 & 1 & 1 & 1 & 1 & 4 \\
\hline Kim et al. [14] & 2012 & 2 & 2 & 0 & 1 & 5 \\
\hline Abdikarim et al. [15] & 2015 & 1 & 1 & 0 & 1 & 3 \\
\hline Liu et al. [16] & 2016 & 2 & 1 & 0 & 1 & 4 \\
\hline Fang et al. [17] & 2016 & 1 & 1 & 0 & 1 & 3 \\
\hline Xia et al. [18] & 2016 & 2 & 2 & 0 & 1 & 5 \\
\hline
\end{tabular}




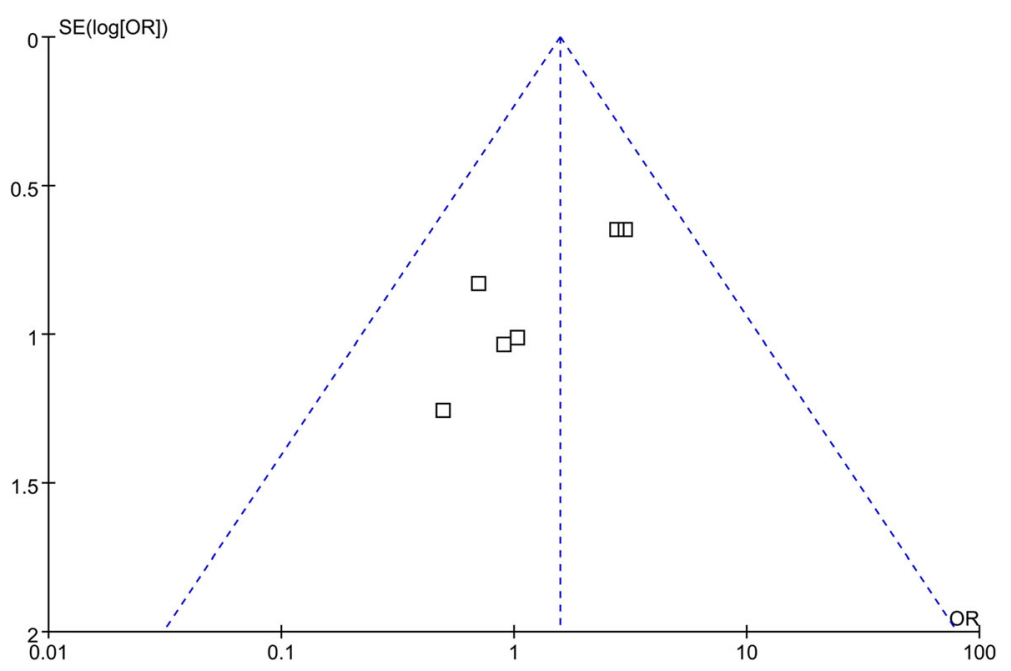

Fig. 2 Funnel plot showing no significant publication bias

conventional care group. The outcome variables (postoperative hospital stay, duration to first flatus after surgery, hospitalization expenditure, and short-term complication) extracted from these studies are presented in Table 2. Two studies did not report duration to first flatus after surgery, and three studies did not report hospital charge $[15,17,18]$.

\section{Methodological quality of included literature}

Regarding the methodological quality of the literature, all six studies were moderate to good (mean quality score 4) (Table 3). Randomization methods were reported in all six trials. Because of the design of the FTS program, most studies did not use blinding methods, except one study which used blinding of assessors [13]. No publication bias between the studies was observed (Fig. 2).

\section{Postoperative hospital stay}

All six studies reported postoperative hospital stay [13-18]. One study reported medians and ranges instead of means and standard deviation (SDs) [13]. So, it could not be used in the meta-analysis. There was significant heterogeneity among the studies $\left(I^{2}=91 \%, P<0.01\right)$ [14-18]. In random effects models, patients in the FTS group had a postoperative hospital stay of 2.65 days less than those in the conventional group (WMD $-2.65 ; 95 \% \mathrm{CI},-4.01$ to $-1.29, z=3.82, P<0.01$; Fig. 3 ).

\section{Duration to first flatus after surgery}

Only three studies reported duration to first flatus after surgery $[14,16,17]$. There was significant heterogeneity among the studies $\left(I^{2}=90 \%, P<0.01\right)$. In random effects models, there was no significant difference in duration to first flatus after surgery between that of the FTS group and conventional group (WMD - 17.72; 95\% CI, - 39.46-4.02, $z=1.60, P=0.11$; Fig. 4).

\section{Hospital charge}

Only three studies reported hospital charge $[13,14,16]$. There was significant heterogeneity among the studies $\left(I^{2}=64 \%, P=0.06\right)$. In random effects models, patients in the FTS group had a hospital charge of 523.43 dollars less

\begin{tabular}{|c|c|c|c|c|c|c|c|c|c|c|c|}
\hline \multirow[b]{2}{*}{ Study or Subgroup } & \multicolumn{3}{|c|}{ Fast-track } & \multicolumn{3}{|c|}{ Conventional } & \multicolumn{2}{|r|}{ Mean Difference } & \multirow{2}{*}{\multicolumn{2}{|c|}{$\begin{array}{c}\text { Mean Difference } \\
\text { IV. Random. } 95 \% \mathrm{Cl}\end{array}$}} & \\
\hline & Mean & SD & Total & Mean & SD & Total & Weight & IV. Random, $95 \% \mathrm{Cl}$ Year & & & \\
\hline Kim JW 2012 & 5.36 & 1.46 & 22 & 7.95 & 1.98 & 22 & $20.5 \%$ & $-2.59[-3.62,-1.56] 2012$ & - & & \\
\hline Abdikarim 2015 & 6.8 & 1.1 & 30 & 7.7 & 1.1 & 31 & $22.4 \%$ & $-0.90[-1.45,-0.35] 2015$ & - & & \\
\hline Xia MJ 2016 & 6.38 & 2.04 & 73 & 8.62 & 2.87 & 76 & $21.5 \%$ & $-2.24[-3.04,-1.44] 2016$ & $\mp$ & & \\
\hline LIU GZ 2016 & 6.3 & 1.5 & 21 & 7.8 & 1.8 & 21 & $20.6 \%$ & $-1.50[-2.50,-0.50] 2016$ & - & & \\
\hline Fang F 2016 & 11 & 2 & 33 & 18.5 & 5.5 & 30 & $15.0 \%$ & $-7.50[-9.58,-5.42] 2016$ & & & \\
\hline Total $(95 \% \mathrm{Cl})$ & & & 179 & & & 180 & $100.0 \%$ & $-2.65[-4.01,-1.29]$ & & & \\
\hline $\begin{array}{l}\text { Heterogeneity: } \text { Tau }^{2} \\
\text { Test for overall effect }\end{array}$ & $\begin{array}{l}2.07 ; C h \\
Z=3.82\end{array}$ & $\begin{array}{l}h i^{2}=42 \\
2(P=0\end{array}$ & $\begin{array}{l}.62, \mathrm{df} \\
.0001)\end{array}$ & $=4(P<$ & $<0.000<-2 x-3<1$ & & & & -10 & 0 & 10 \\
\hline
\end{tabular}

Fig. 3 Forest plot describing postoperative hospital stay between FTS and conventional recovery strategies in laparoscopic gastrectomy for gastric cancer. FTS fast-track surgery 


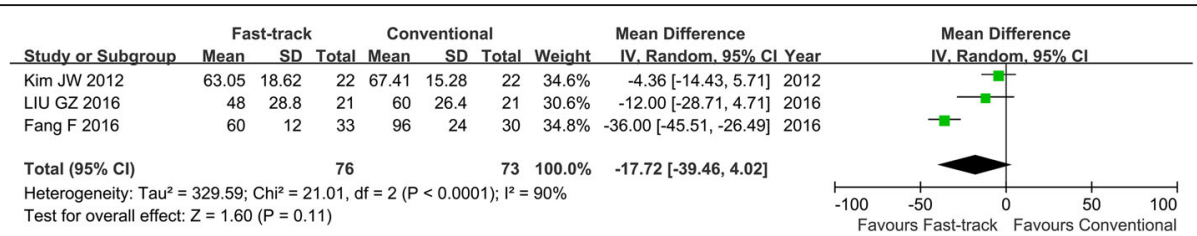

Fig. 4 Forest plot describing duration to first flatus after surgery between FTS and conventional recovery strategies in laparoscopic gastrectomy for gastric cancer. FTS fast-track surgery

than those in the conventional group. (WMD - 523.43; 95\% CI, -799.79 to $-247.06, z=3.71, P<0.01$; Fig. 5).

\section{Overall complication rate}

All six studies reported postoperative complication rate [13-18]. There was no significant heterogeneity among the studies $\left(I^{2}=0 \%, P=0.56\right)$. In fixed effects models, there was no significant difference in complication rates between the FTS group and conventional group. (OR 1.57; 95\% CI, 0.82-2.98, $z=1.37, P=0.17$; Fig. 6).

\section{Discussion}

The fast-track surgery (FTS) consists of a multidisciplinary approach including patients' education, anesthesia, goal-directed fluid therapy, prevention of nausea and ileus, temperature monitoring, early nutrition, and early mobilization. Many studies had demonstrated that FTS in D2 gastrectomy was safe and efficient, and it could reduce postoperative stresses [21]. Some metaanalysis also demonstrated the FTS protocol was feasible for gastric cancer patients who underwent gastrectomy. Li et al. found that compared with conventional care, FTS shortened the postoperative stay (WMD $-2.00 ; 95 \%$ CI -2.69 to $-1.30, z=5.64, P<$ 0.00001 ), and reduced hospitalization costs (WMD 447.72; 95\% CI -615.92 to $-279.51, z=5.22, P<0.00001$ ). There was no significant difference in postoperative complications $(P=0.07)$ [22]. Yu et al. showed that postoperative hospital stay, time to first passage of flatus, and hospital costs were significantly reduced for fast-track surgery. No significant differences were found for readmission rates and total postoperative complications [23]. All these meta-analyses focused on open gastrectomy for gastric cancer combined with FTS protocol. Little study focused on the safety and effectiveness in laparoscopic gastrectomy combined with FTS protocol. Recently, the advantages of laparoscopic gastrectomy had also been recognized. Some studies had demonstrated that laparoscopic gastrectomy could alleviate inflammation and immune inhibition and accelerate postoperative recovery [24-29]. However, there was little study comparing the effect of FTS program in laparoscopic gastrectomy, and the result was conflicting [13-18]. So, in the present study, we focused on four short-term outcomes and aimed to investigate the safety and efficacy of FTS in laparoscopic gastrectomy for gastric carcinoma.

Our study revealed postoperative hospital stay was significantly shorter in the FTS group than in the conventional group (Fig. 3). Both FTS and laparoscopic surgery could reduce the postoperative stress response and promote rehabilitation [30-32]. Therefore, combining the two methods would result in the fastest postoperative recovery. We also found there was a trend toward shorter time of first flatus for the FTS group. However, the result of the meta-analysis failed to show any difference, although the trend favored the FTS group (Fig. 4). The reason might be that the power of the test was weak. The sample size was too small to detect the real difference between the two groups. In our study, the hospital charge was significantly different between the two groups. The FTS group had lower medical costs than conventional group (Fig. 5), which might be explained by the effect of the fasttrack recovery system.

With respect to complication rate, there was no significant difference in complication rates between the FTS group and conventional group (Fig. 6). In the

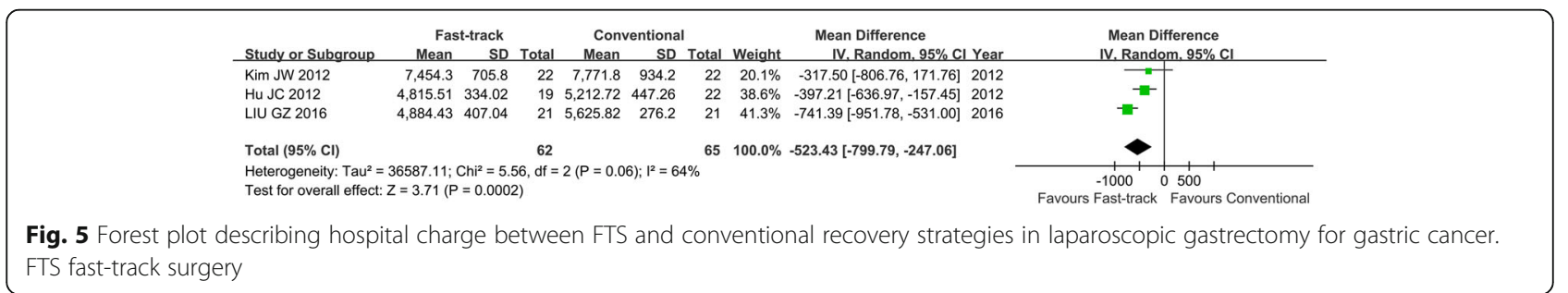




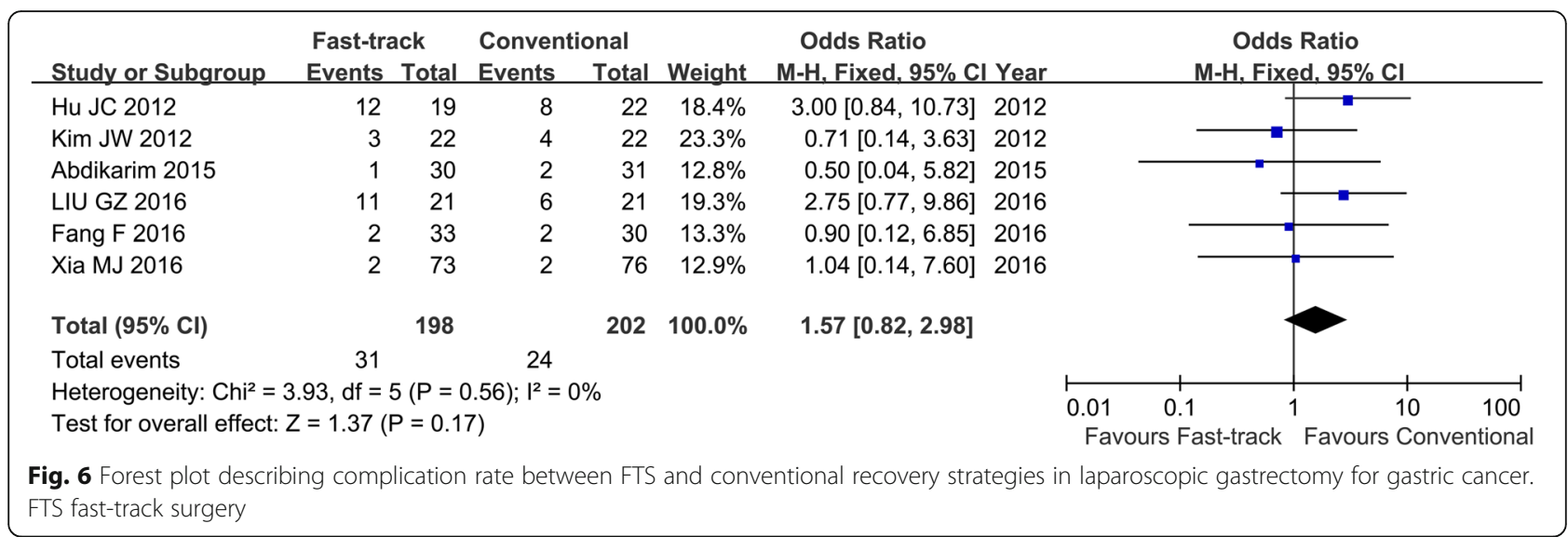

present meta-analysis, the complication rates in each study were varied (2.63-63.2\%). Many factors could influence the occurrence of postoperative complications, like surgeon's surgical technique, perioperative care, and physical condition of patients. However, we found a trend toward higher complication rate for the FTS group in the present study (Fig. 6). We were not sure whether this was associated with perioperative FTS. Therefore, more studies were needed to confirm the safety of FTS in laparoscopic gastrectomy.

There were some limitations to this study. Firstly, the sample of RCTs was small. Therefore, the power of the test might be weakened and the real difference might not be apparent presently. Secondly, the studies showed significant heterogeneity in the outcome measures. This heterogeneity might be due to diversity in medical and economic status in different regions. Thirdly, only two studies included in the metaanalysis dealt solely with laparoscopic distal gastrectomy $[13,14]$. The other four studies dealt with the laparoscopic proximal stomach, distal gastrectomy, and total gastrectomy. It was not possible to tease out these numbers. Therefore, we could not know whether the results will change if the total gastrectomy patients were separated from the distal gastrectomy patients. Last but not least, methodological quality of included studies was moderate. Most studies did not use blinding methods, except one study which used blinding of assessors. It was hard to use double-blind methods in most clinical trials, and therefore, this limitation was difficult to solve.

\section{Conclusions}

This meta-analysis showed that fast-track perioperative care has shorter postoperative hospital stays and less hospitalization expenditure than conventional recovery strategies in laparoscopic gastrectomy. There is no significant difference with respect to complication rate. Fast-track surgery is safe and effective in laparoscopic gastrectomy for gastric carcinoma. However, the sample of RCTs included in the meta-analysis is small. Large-sample randomized controlled trials should be encouraged to ensure that the benefits of fast-track surgery can be applied to laparoscopic gastrectomy.

\section{Abbreviations}

Cl: Confidence intervals; ERAS: Enhanced recovery after surgery; FTS: Fasttrack surgery; OR: Odds ratio; PRISMA: Preferred Reporting Items for Systematic Reviews and Meta-Analyses WMDWeighted mean difference; RCTs: Randomized controlled trials; SD: Standard deviation

\section{Acknowledgements}

Not applicable

\section{Funding}

This research did not receive any specific grant from funding agencies in the public, commercial, or not-for-profit sectors.

Availability of data and materials

The datasets generated and/or analyzed during the current study are available in the Pubmed, Medline, Embase, and Cochrane library databases.

\section{Authors' contributions}

$M L, W W, J L$, and $Y H$ were in charge of designing, drafting, and revising the article and approving the final version to be published. $L L$ and $X Z$ were in charge of designing, revising the article critically, and approving the final version to be published. $\mathrm{HZ}$ was in charge of analyzing and interpreting of data, drafting the article, and approving the final version to be published. All authors read and approved the final manuscript.

\section{Ethics approval and consent to participate}

This study was approved by the ethic committee of the Seventh Affiliated Hospital of Sun Yat-sen University.

\section{Consent for publication}

Not applicable

\section{Competing interests}

The authors declare that they have no competing interests.

\section{Publisher's Note}

Springer Nature remains neutral with regard to jurisdictional claims in published maps and institutional affiliations. 
Received: 2 August 2017 Accepted: 8 January 2018

Published online: 26 January 2018

\section{References}

1. Price TJ, Shapiro JD, Segelov E, Karapetis CS, Pavlakis N, Van Cutsem E, et al. Management of advanced gastric cancer. Expert Rev Gastroenterol Hepatol. 2012:6:199-208.

2. Jeong SH, Lee YJ, Park ST, Choi SK, Hong SC, Jung EJ, et al. Risk of recurrence after laparoscopy-assisted radical gastrectomy for gastric cancer performed by a single surgeon. Surg Endosc. 2011;25:872-8.

3. Kim YW, Baik YH, Yun YH, Nam BH, Kim DH, Choi IJ, et al. Improved quality of life outcomes after laparoscopy-assisted distal gastrectomy for early gastric cancer: results of a prospective randomized clinical trial. Ann Surg. 2008;248:721-7.

4. Adachi $Y$, Suematsu T, Shiraishi N, Katsuta T, Morimoto A, Kitano S, et al. Quality of life after laparoscopy-assisted Billroth I gastrectomy. Ann Surg. 1999;229:49-54

5. Bardram L, Funch-Jensen $P$, Jensen $P$, Crawford ME, Kehlet H. Recovery after laparoscopic colonic surgery with epidural analgesia, and early oral nutrition and mobilisation. Lancet. 1995:345:763-4.

6. D'Hondt M, Vanneste S, Pottel H, Devriendt D, Van Rooy F, Vansteenkiste F. Laparoscopic sleeve gastrectomy as a single-stage procedure for the treatment of morbid obesity and the resulting quality of life, resolution of comorbidities, food tolerance, and 6-year weight loss. Surg Endosc. 2011;25: 2498-504

7. Liu XX, Jiang ZW, Wang ZM, Li JS. Multimodal optimization of surgical care shows beneficial outcome in gastrectomy surgery. J Parenter Enter Nutr. 2010;34:313-21.

8. Zouros E, Liakakos T, Machairas A, Patapis P, Agalianos C, Dervenis C. Improvement of gastric emptying by enhanced recovery after pancreaticoduodenectomy. Hepatobiliary Pancreat Dis Int. 2016;15:198-208.

9. Wang D, Kong Y, Zhong B, Zhou X, Zhou Y. Fast-track surgery improves postoperative recovery in patients with gastric cancer: a randomized comparison with conventional postoperative care. J Gastrointest Surg. 2010; 14:620-7.

10. Feng F, Ji G, Li JP, Li XH, Shi H, Zhao ZW, et al. Fast-track surgery could improve postoperative recovery in radical total gastrectomy patients. World J Gastroenterol. 2013;19:3642-8.

11. Yamada T, Hayashi T, Cho H, Yoshikawa T, Taniguchi H, Fukushima R, et al. Usefulness of enhanced recovery after surgery protocol as compared with conventional perioperative care in gastric surgery. Gastric Cancer. 2012;15:34-41.

12. Liu XX, Pan HF, Jiang ZW, Zhang S, Wang ZM, Chen P, et al. "Fast-track" and "minimally invasive" surgery for gastric cancer. Chin Med J. 2016;129:2294-300.

13. Hu JC, Jiang LX, Cai L, Zheng HT, Hu SY, Chen HB, et al. Preliminary experience of fast-track surgery combined with laparoscopy-assisted radical distal gastrectomy for gastric cancer. J Gastrointest Surg. 2012;16:1830-9.

14. Kim JW, Kim WS, Cheong JH, Hyung WJ, Choi SH, Noh SH. Safety and efficacy of fast-track surgery in laparoscopic distal gastrectomy for gastric cancer: a randomized clinical trial. World J Surg. 2012;36:2879-87.

15. Abdikarim I, Cao XY, Li SZ, Zhao YQ, Taupyk Y, Wang Q. Enhanced recovery after surgery with laparoscopic radical gastrectomy for stomach carcinomas. World J Gastroenterol. 2015;21:13339-44.

16. Liu G, Jian F, Wang $X$, Chen L. Fast-track surgery protocol in elderly patients undergoing laparoscopic radical gastrectomy for gastric cancer: a randomized controlled trial. Onco Targets Ther. 2016:9:3345-51.

17. Fang F, Gao J, Bi X, Han F, Wang HJ. Effect and clinical significance of fasttrack surgery combined with laparoscopic radical gastrectomy on the plasma level of vascular endothelial growth factor in gastric antrum cancer. Spring. 2016;5:50.

18. Xia M, Zhang L, Tang Z, Zhao Y, Wang Q. Laparoscopic radical gastrectomy for resectable advanced gastric cancer within enhanced recovery programs: a prospective randomized controlled trial. J Laparoendosc Adv Surg Tech A. 2016. PMID: 27875094; https://doi.org/10.1089/lap.2016.0057.

19. Moher D, Liberati A, Tetzlaff J, Altman DG, PRISMA Group. Preferred reporting items for systematic reviews and meta-analyses: the PRISMA statement. Int J Surg. 2010;8:336-41.

20. Jadad AR, Moore RA, Carroll D, Jenkinson C, Reynolds DJ, Gavaghan DJ, et al. Assessing the quality of reports of randomized clinical trials: is blinding necessary? Control Clin Trials. 1996;17:1-12.
21. Bu J, Li N, Huang X, He S, Wen J, Wu X. Feasibility of fast-track surgery in elderly patients with gastric cancer. J Gastrointest Surg. 2015;19:1391-8.

22. Li YJ, Huo TT, Xing J, An JZ, Han ZY, Liu XN, et al. Meta-analysis of efficacy and safety of fast-track surgery in gastrectomy for gastric cancer. World J Surg. 2014;38(12):3142-51.

23. Yu Z, Zhuang CL, Ye XZ, Zhang CJ, Dong QT, Chen BC. Fast-track surgery in gastrectomy for gastric cancer: a systematic review and meta-analysis. Langenbeck's Arch Surg. 2014;399(1):85-92.

24. Nishiguchi K, Okuda J, Toyoda M, Tanaka K, Tanigawa N. Comparative evaluation of surgical stress of laparoscopic and open surgeries for colorectal carcinoma. Dis Colon Rectum. 2001:44:223-30.

25. Yu G, Tang B, PW Y, Peng ZH, Qian F, Sun G. Systemic and peritoneal inflammatory response after laparoscopic-assisted gastrectomy and the effect of inflammatory cytokines on adhesion of gastric cancer cells to peritoneal mesothelial cells. Surg Endosc. 2010;24:2860-70.

26. Lee JH, Yom CK, Han HS. Comparison of long-term outcomes of laparoscopy-assisted and open distal gastrectomy for early gastric cancer. Surg Endosc. 2009;23:1759-63.

27. Etoh T, Shiraishi N, Kitano S. Laparoscopic gastrectomy for cancer. Dig Dis. 2005:23:113-8.

28. Grantcharov TP, Kehlet H. Laparoscopic gastric surgery in an enhanced recovery programme. Br J Surg. 2010:97:1547-51.

29. Lee $\mathrm{JH}, \mathrm{Han} \mathrm{HS}$. A prospective randomized study comparing open vs laparoscopy-assisted distal gastrectomy in early gastric cancer: early results. Surg Endosc. 2005;19:168-73.

30. Jiang ZW, Zhang S, Wang G, Zhao K, Liu J, Ning L, et al. Single-incision laparoscopic distal gastrectomy for early gastric cancer through a homemade single port access device. Hepato-Gastroenterology. 2015;62: $518-23$

31. Chen S, Zou Z, Chen F, Huang Z, Li G. A meta-analysis of fast track surgery for patients with gastric cancer undergoing gastrectomy. Ann R Coll Surg Engl. 2015;97:3-10.

32. Aoyama T, Yoshikawa T, Hayashi T, Hasegawa S, Tsuchida K, Yamada T, et al. Randomized comparison of surgical stress and the nutritional status between laparoscopy-assisted and open distal gastrectomy for gastric cancer. Ann Surg Oncol. 2014;21:1983-90.

\section{Submit your next manuscript to BioMed Central and we will help you at every step:}

- We accept pre-submission inquiries

- Our selector tool helps you to find the most relevant journal

- We provide round the clock customer support

- Convenient online submission

- Thorough peer review

- Inclusion in PubMed and all major indexing services

- Maximum visibility for your research

Submit your manuscript at www.biomedcentral.com/submit

) BioMed Central 